\title{
SAND98-1295C
}

\section{Wavelet Compression of Complex SAR Imagery Using Complex- and Real- Valued Wavelets: A Comparative Study}

\author{
LCDR R.W. Ives, USN ${ }^{1}$, N. Magotra ${ }^{2}$ and C. Kiser $^{3}$ \\ ${ }^{1}$ Sandia National Laboratories, Albuquerque, New Mexico, USA 87185 \\ ${ }^{2}$ EECE Department, University of New Mexico, Albuquerque, New Mexico, USA 87131 \\ ${ }^{3}$ Aquila Technologies Group, Inc., Albuquerque, NM, USA 87113
}

\begin{abstract}
While many synthetic aperture radar (SAR) applications use only detected imagery, dramatic improvements in resolution and employment of algorithms requiring complex-valued SAR imagery suggest the need for compression of complex data. Here, we investigate the benefits of using complexvalued wavelets on complex SAR imagery in the embedded zerotree wavelet compression algorithm, compared to using real-valued wavelets applied separately to the real and imaginary components. This compression is applied at low ratios $(4: 1-12: 1)$ for high fidelity output. The complex spatial correlation metric is used to numerically evaluate quality. Numerical results are tabulated and original and decompressed imagery are presented as well as correlation maps to allow visual comparisions.
\end{abstract}

\section{Introduction}

The immediate goal of this research is to address the problem of transmitting massive amounts of high resolution complex SAR data from a remote airborne sensor to a ground station for exploitation in real-time. This data may be used for interferometry, coherent change detection or some other application in which complex-valued data is required. However, the quality of the data must be sufficiently maintained to allow little or no impact on the application if any loss of information is incurred in its compression.

Wavelets are widely used in lossy image compression schemes for their ease of implementation and performance. Since applications which use realvalued data in general are more common (such as those which require photographic imagery, detected or logmapped radar imagery, etc.), a great deal of available research has been applied to the use of real-valued wavelet filter banks on real-valued data. This paper addresses the application of a wavelet compression scheme to complex-valued imagery. The method of compression upon which this research is based is the embedded zerotree wavelet (EZW) coding algorithm of Shapiro, described in [1]. The question we sought to answer is: "Is there an advantage to using complexvalued wavelets in compression on complex imagery ?"

The data used in this research is both one-meter and one-foot resolution complex imagery collected by the Sandia National Laboratories' SAR carried onboard the U.S. Department of Energy's Twin Otter aircraft. These images are urban and rural scenes of areas on Kirtland Air Force Base in Albuquerque. Each pixel has a 16-bit signed integer real and imaginary component (32bits/pixel).

\section{Methodology}

To apply real-valued wavelets, each SAR complex image was first segregated into real and imaginary "images". For each, the wavelet transform produced a real-valued coefficient image, which was then compressed using the EZW algorithm. These two coefficient images were compressed independently at the encoder and decompressed independently at the decoder. After decompression and the inverse wavelet transforms, the two reconstructed real and imaginary "images" were formed into a lossy decompressed version of the original complex image.

Using complex-valued wavelets directly on complex imagery, the wavelet transform produces a complex coefficient "image". To apply the EZW algorithm in this case, the complex coefficient image was segregated into its real and imaginary components. The zerotree coding was then applied to each component "image" independently. The decoder performed the reverse operation: zerotree decoding the real and imaginary coefficient images independently which are then combined into a complex wavelet transformed image. 


\section{DISCLAIMER}

This report was prepared as an account of work sponsored by an agency of the United States Government. Neither the United States Government nor any agency thereof, nor any of their employees, make any warranty, express or implied, or assumes any legal liability or responsibility for the accuracy, completeness, or usefulness of any information, apparatus, product, or process disclosed, or represents that its use would not infringe privately owned rights. Reference herein to any specific commercial product, process, or service by trade name, trademark, manufacturer, or otherwise does not necessarily constitute or imply its endorsement, recommendation, or favoring by the United States Government or any agency thereof. The views and opinions of authors expressed herein do not necessarily state or reflect those of the United States Government or any agency thereof. 


\section{DISCLAIMER}

\section{Portions of this document may be illegible in electronic image products. Images are produced from the best available original document.}


The complex inverse wavelet transform then generated a lossy decompressed version of the original image.

The real-valued wavelets used in testing include the Daubechies 4, 6, 8 and 10-tap orthogonal filters, the Haar filter, and the biorthogonal $6 / 6,9 / 7,5 / 3,2 / 6$ and $9 / 3$ filter banks. The complex-valued filters used were the normal symmetric complex Daubechies 10 and 6-tap filters, and four of the conjugate quadrature 4-tap filters described in [2]. In addition, a length two complex conjugate symmetric filter bank was employed. This filter bank was derived using a construction procedure contained in [3]. For all filters, a range of compression ratios was tested from 4:1 to $12: 1$ in increments of 2 . A more detailed analysis of SAR image compression using real-valued wavelets (on both detected and complex SAR imagery) may be found in [4].

\section{Quality metric}

Various measures have been used to quantify the quality of lossy compressed imagery, including SNR and MSE. But these metrics have been shown to provide misleading results particularly with large dynamic range data such as SAR [4]. In the case at hand, the object is to determine the quality degradation between two complex SAR images which are of the same size and are registered, since one is just a compressed/decompressed version of the other. From these two images a correlation map may be computed. This map will have the same dimensions as the original: for each pixel location, the correlation coefficient is calculated based on the pixels in a small neighborhood about it ( $5 \times 5$ for example) using common pixels in each image. In this manner, the sample complex spatial correlation coefficient for a pixel at location $(x, y)$ between an original and decompressed SAR image can be computed from [5]:

$$
c(x, y)=\frac{\left|\sum_{i} \sum_{j} f(i, j) \cdot g^{*}(i, j)\right|}{\sqrt{\sum_{i} \sum_{j}\|f(i, j)\|^{2} \cdot \sum_{i} \sum_{j}\|g(i, j)\|^{2}}}
$$

where the indices $i$ and $j$ represent pixels in a neighborhood about pixel $(x, y)$, and $f$ and $g$ are the original and decompressed images. This correlation is measured over a neighborhood about each pixel in the chip, and will fall in the range [0.0,1.0]. Perfect correlation for a particular pixel is indicated by a value of 1.0 and occurs if every complex value within the neighborhood of the original pixel is equal to the corresponding pixel value in the decompressed neighborhood: a value of 0.0 indicates that they are completely uncorrelated. Equation (1) assumes a zero mean in the neighborhood, which is generally true for complex SAR images with sufficient neighborhood size.

This correlation map may be viewed to subjectively judge the quality of the decompressed image as a whole or to see where the areas of highest/lowest correlation are. Additionally, this correlation metric leads to a single global quality measure, the average spatial correlation, which is just the mean value of the correlation map. This metric has been shown to be more robust than MSE and SNR and more consistent with visual quality [4].

\section{Results and conclusions}

Table 1 summarizes the overall correlation results for six complex Twin-Otter images. Each interior entry of the table represents the computed average spatial correlation of each image at the various compression ratios, averaged over all six images.

\begin{tabular}{|c|c|c|c|c|c|}
\hline \multicolumn{6}{|c|}{ Real-Valued Wavelets } \\
\hline & $4: 1$ & $6: 1$ & $8: 1$ & $10: 1$ & $12: 1$ \\
\hline Haar & 0.96 & 0.94 & 0.91 & 0.88 & 0.86 \\
\hline Daubechies 4-tap & .96 & 0.94 & 0.92 & 0.90 & 0.89 \\
\hline Daubechies 6-tap & 96 & 0.94 & 0.93 & 0.91 & 0.89 \\
\hline Daubechies 8-tap & 0.95 & 0.94 & 0.93 & 0.91 & 0.90 \\
\hline Daubechies 10-tap & 0.95 & 0.94 & 0.93 & 0.91 & 0.91 \\
\hline Biorth & 0.96 & 0.95 & 0.93 & 0.91 & 0.90 \\
\hline Biorth & 0.95 & 0.95 & 0.93 & 0.92 & 0.91 \\
\hline Biortho & 0.95 & 0.94 & 0.93 & 0.91 & 0.89 \\
\hline Biorthogonal $2 / 6$ & 0.96 & 0.95 & 0.93 & 0.91 & 0.90 \\
\hline Biorthogonal 9/3 & 0.95 & 0.94 & 0.92 & 0.91 & 0.90 \\
\hline \multicolumn{6}{|c|}{ Complex-Valued Wavelets } \\
\hline & $4: 1$ & $6: 1$ & $8: 1$ & $10: 1$ & $12: 1$ \\
\hline Com & 0.96 & 0.94 & 0.90 & 0.88 & 0.86 \\
\hline $\begin{array}{l}\text { Compl } \\
\left(\varphi_{1}=-\right.\end{array}$ & 0.96 & 0.94 & 0.91 & 0.89 & 0.88 \\
\hline $\begin{array}{l}\text { Complex 4-tap } \\
\left(\varphi_{1}=-\pi / 4\right)\end{array}$ & 0.95 & 0.92 & 0.90 & 0.87 & 0.84 \\
\hline $\begin{array}{l}\text { Complex 4-tap } \\
\left(\varphi_{1}=-3 \pi / 8\right)\end{array}$ & 0.95 & 0.91 & 0.87 & 0.84 & 0.81 \\
\hline $\begin{array}{l}\text { Complex 4-tap } \\
\left(\varphi_{1}=-\pi / 2\right)\end{array}$ & 0.94 & 0.90 & 0.86 & 0.82 & 0.79 \\
\hline $\begin{array}{l}\text { Complex } \\
\text { Daubechies 6-tap }\end{array}$ & 0.95 & 0.95 & 0.93 & 0.91 & 0.89 \\
\hline $\begin{array}{l}\text { Complex } \\
\text { Daubechies 10-tap }\end{array}$ & 0.95 & 0.94 & 0.92 & 0.91 & 0.89 \\
\hline
\end{tabular}

Table 1: Mean Values of the Average Spatial Correlation Over All Imagery

From the above table, several points become clear. Primarily we notice that there was definitely no 
advantage to using complex-valued wavelets, at least not so for those wavelets tested. In fact, in almost all cases, real-valued wavelets outperformed the complexvalued wavelets at every compression ratio. Secondly, we note that the quality using complex wavelets dropped off with increasing compression ratio faster than the real wavelets. Finally, we note that the use of complex wavelets involves a greater computational burden than using real wavelets on the real and imaginary planes separately. For example, consider a 2tap filter bank to compute one complex wavelet coefficient: using the Haar wavelet on the two planes separately requires a total of four multiplies and two additions, while using a 2-tap complex wavelet requires eight multiplies and six adds, due to the cross terms.

We attribute these spatial correlation results to the large presence of distributed targets throughout SAR imagery (natural scatterers such as grass, water, rocks, etc.). The in-phase and quadrature components of these complex pixel values can be modeled as samples from zero-mean, independent and identically distributed gaussian probability densities [6] and as such offer no correlation between the real and imaginary planes. This fact is borne out if the correlation coefficient [7] is computed for each pixel between the original real "image" and the imaginary "image". This value, which will fall in the range [-1.0,1.0], with 1.0 indicating strong correlation, -1.0 indicating strong negative correlation and 0.0 representing no correlation, will yield an average correlation close to zero when applied to the component planes of a complex SAR image. Complex wavelets, then, have no correlation to take

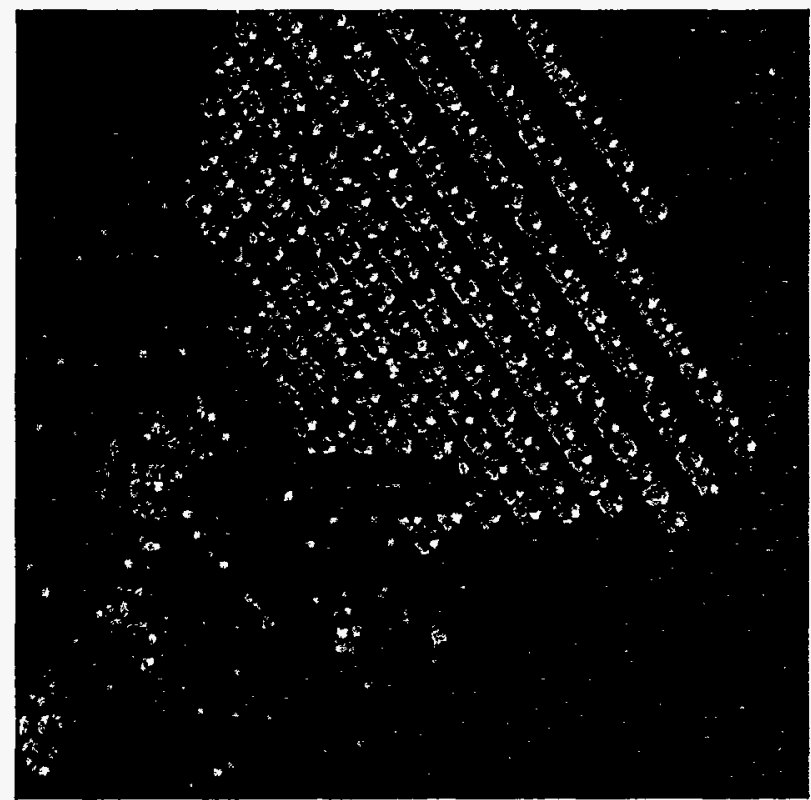

Figure 2: Solar.cmpx (512x512, $1 \mathrm{~m}$ res) advantage of between the planes in these areas of the image. Real wavelets however, are better suited to take advantage of whatever spatial correlation exists within either the real or imaginary plane.

As an example of correlation maps between original and decompressed imagery, a complex image of the Sandia Solar Test Facility collected by the Sandia Otter platform was compressed at 6:1 and 12:1 (using the biorthogonal $2 / 6$ real-valued wavelet filters separately on the real and imaginary components). Figure 1 is a detected and log-mapped version of the original image.

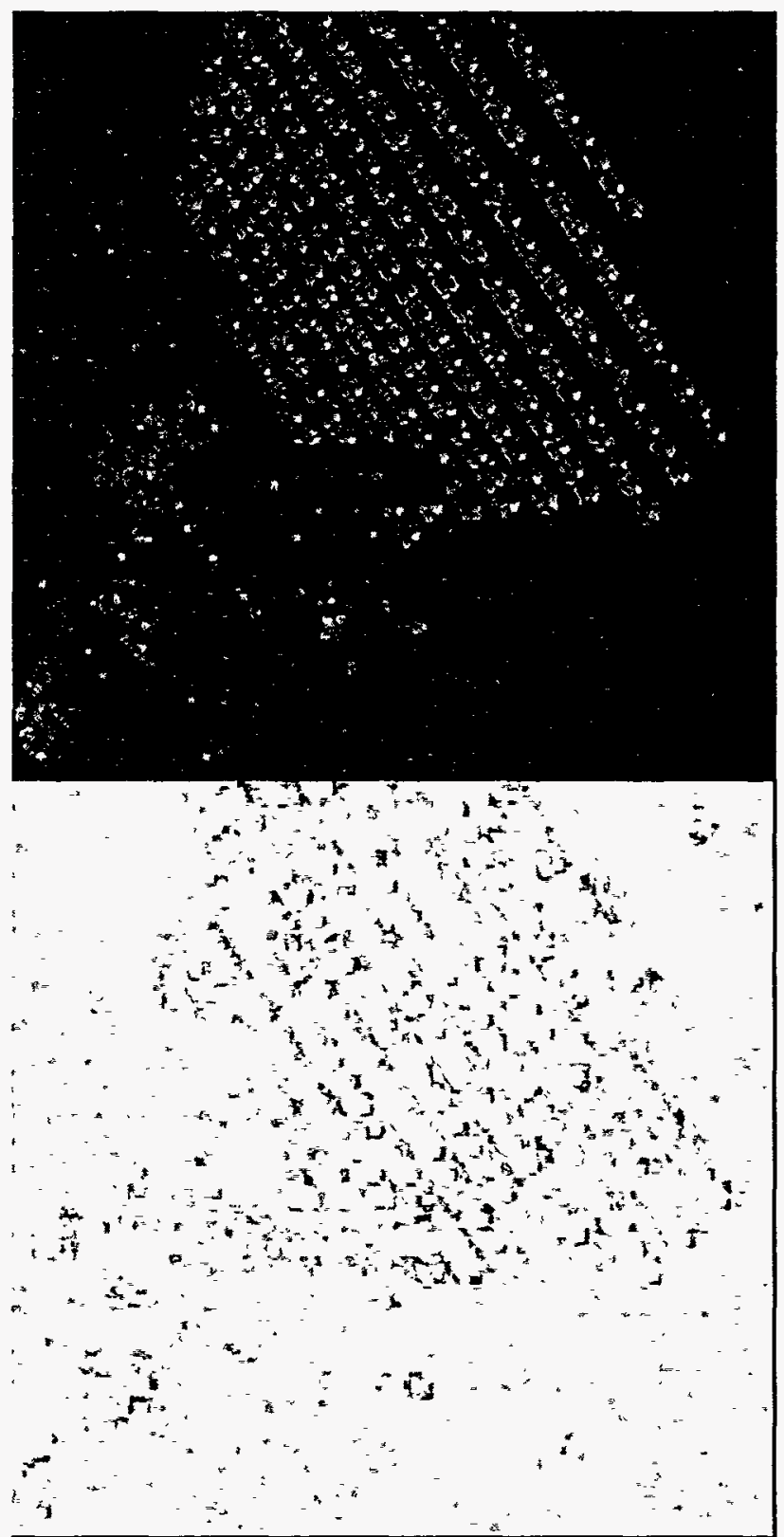

Figure 1: Decompressed at 6:1 (top) and Correlation Map (bottom) 
Figure 2 shows the decompressed result at a 6:1 compresson ratio (top) and the resulting complex correlation map (bottom). This map was formed with a $5 \times 5$ correlation neighborhood, and the mean value of this correlation map, the average spatial correlation has a value of 0.95 . Figure 3 shows the decompressed result at a compression ratio of 12:1 (top) and the corresponding correlation map (bottom). In this figure, the average spatial correlation is 0.87 . The spatial correlation map is brighter overall for the $6: 1$ compression, corresponding to a higher quality than the

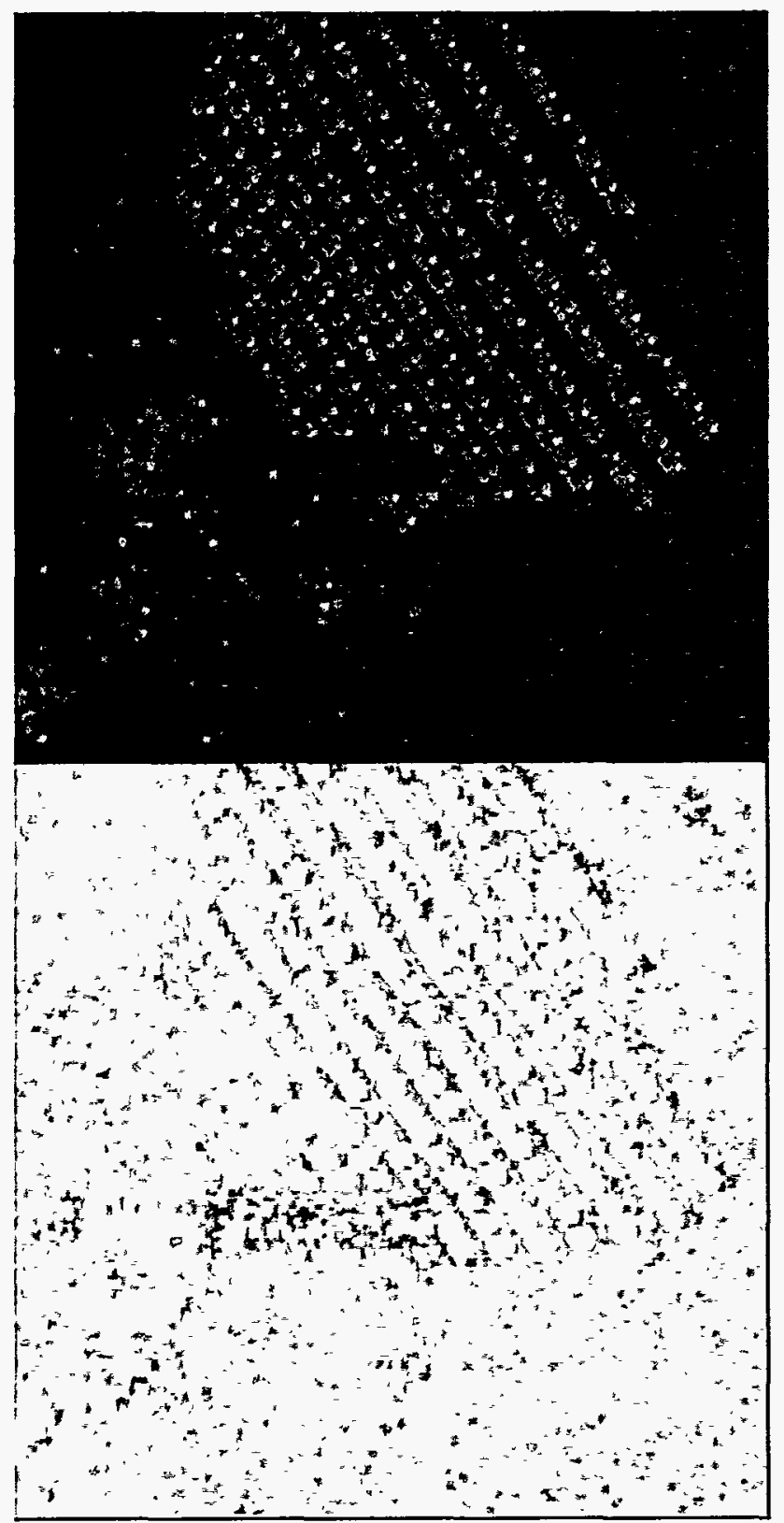

Figure 3: Decompressed at 12:1 (top) and Correlation Map (bottom)
12:1 image. Visually comparing the decompressed imagery, the differences can be seen (after detection) as blurring of point targets, increased noise in shadows and some smoothing around edges. This overall quality difference is also demonstrated in the values of the average spatial correlation for the two decompressed images ( 0.95 vs. 0.87 ).

Figures 4 through 8 are included to display the other five complex images used in this research. The size and resolution of each image is included.

\section{Acknowledgements}

Sandia is a multiprogram laboratory operated by Sandia Corporation, a Lockheed Martin Company, for the United States Department of Energy under contract DE-AC04-94AL85000.

\section{References}

[1] J. Shapiro, "Embedded image coding using zerotrees of wavelet coefficients", IEEE Transactions on Signal Processing, Vol. 41, No. 12, pp. 3445-3462, Dec. 1993.

[2] Zhang, X, Desai, M. and Peng, Y., "Orthogonal Complex Filter Banks and Wavelets: Some Properties and Design", to. appear in IEEE Transactions on Signal Processing.

[3] Frazier, M. An Introduction to Wavelets Through Linear Algebra. Preprint, Department of Mathematics, Michigan State University.

[4] R. Ives, On the Compression of Synthetic Aperture Radar Imagery, PhD Dissertation, University of New

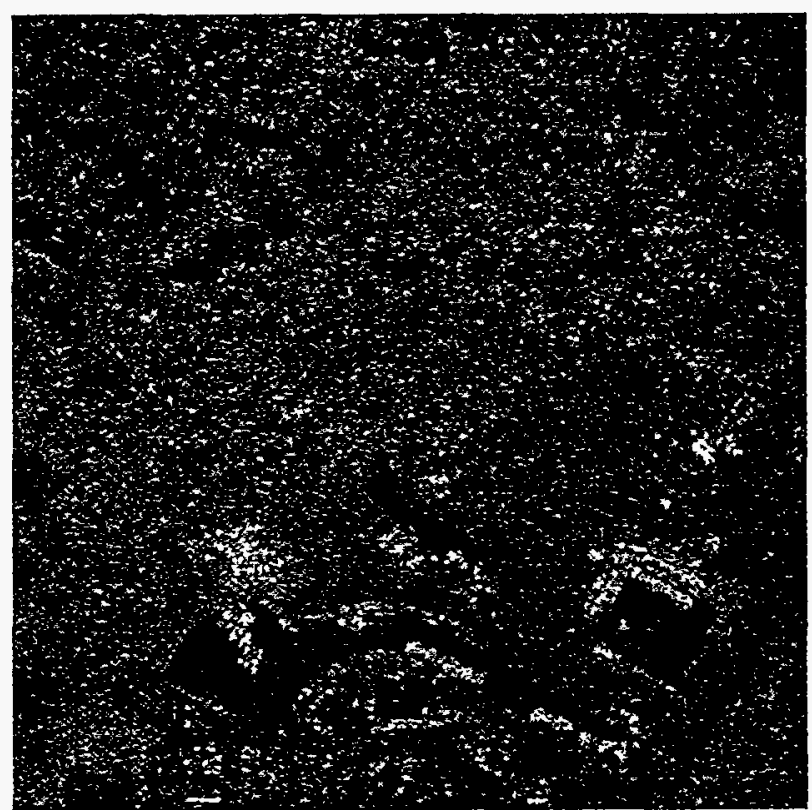

Figure 4: Buildings.cmpx (1024x1024, $1 \mathrm{~m}$ res) 
Mexico, May, 1998.

[5] C. Jakowatz et al, Spotlight-Mode Synthetic Aperture Radar: A Signal Processing Approach, Kluwer Academic Publishers, Boston, MA, 1996.

[6] J. Curlander and R. McDonough. Synthetic Aperture Radar: Systems \& Signal Processing. John Wiley \& Sons, Inc.: New York, 1991.

[7] .R. Gonzalez and R. Woods, Digital Image Processing. Addison-Wesley Publishing Co., Inc.: Reading, MA, 1992.

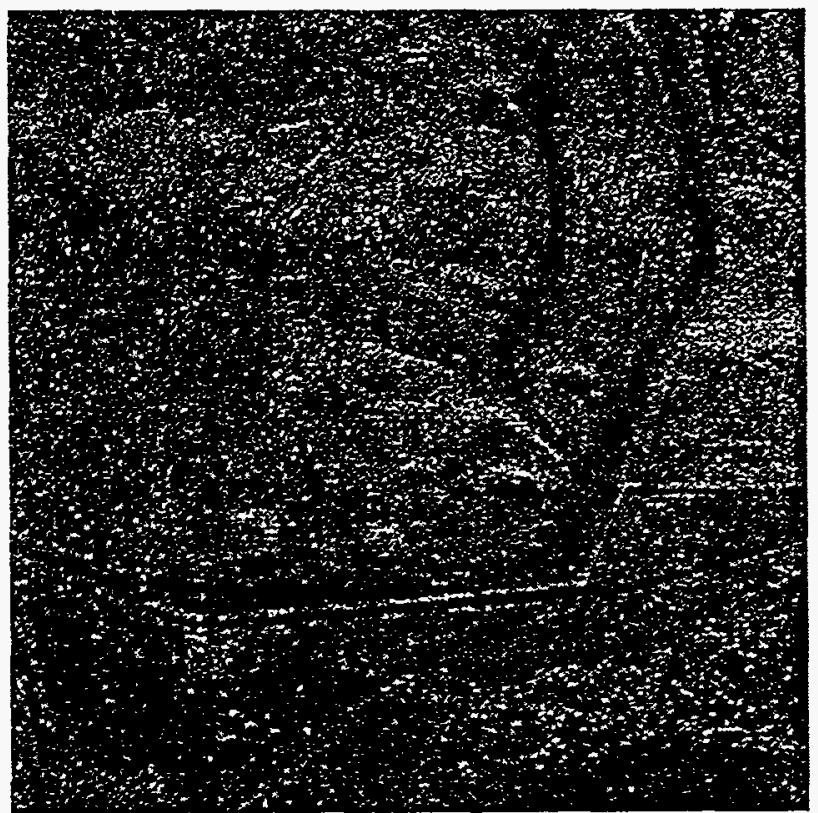

Figure 5: Bunkers.cmpx (1600x1600, $1 \mathrm{ft}$. res)

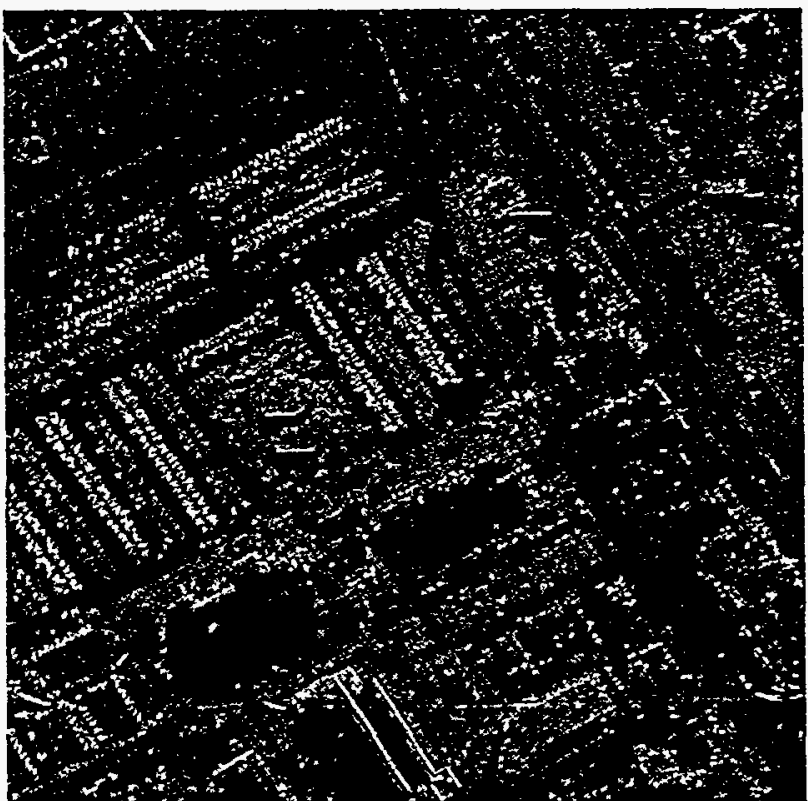

Figure 6: City.cmpx (1600x1600, $1 \mathrm{ft}$ res)

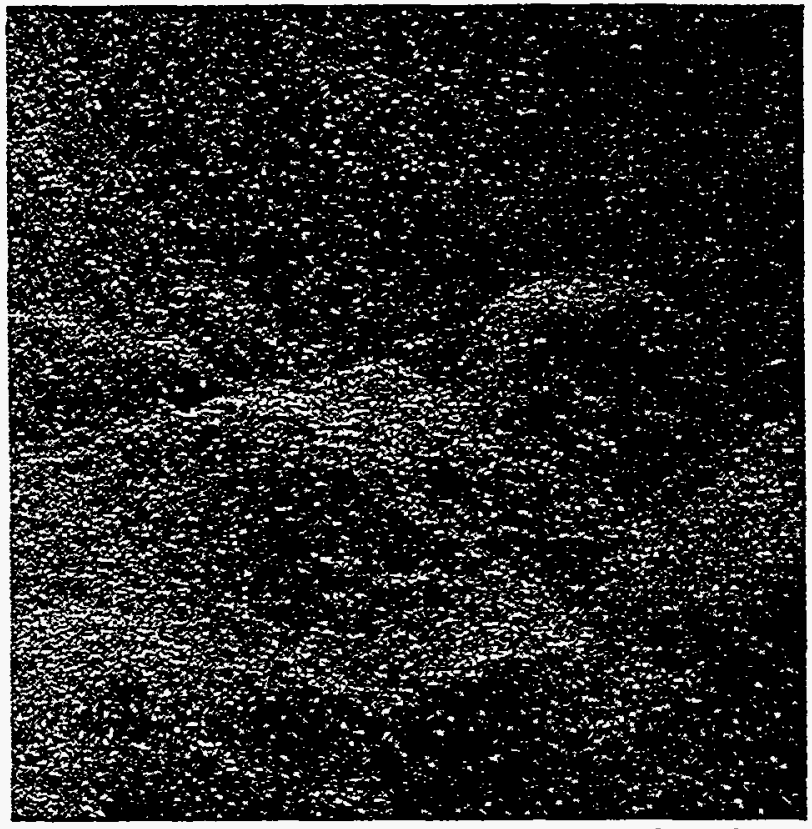

Figure 7: Hills.cmpx (1600x1600, $1 \mathrm{ft}$ res)

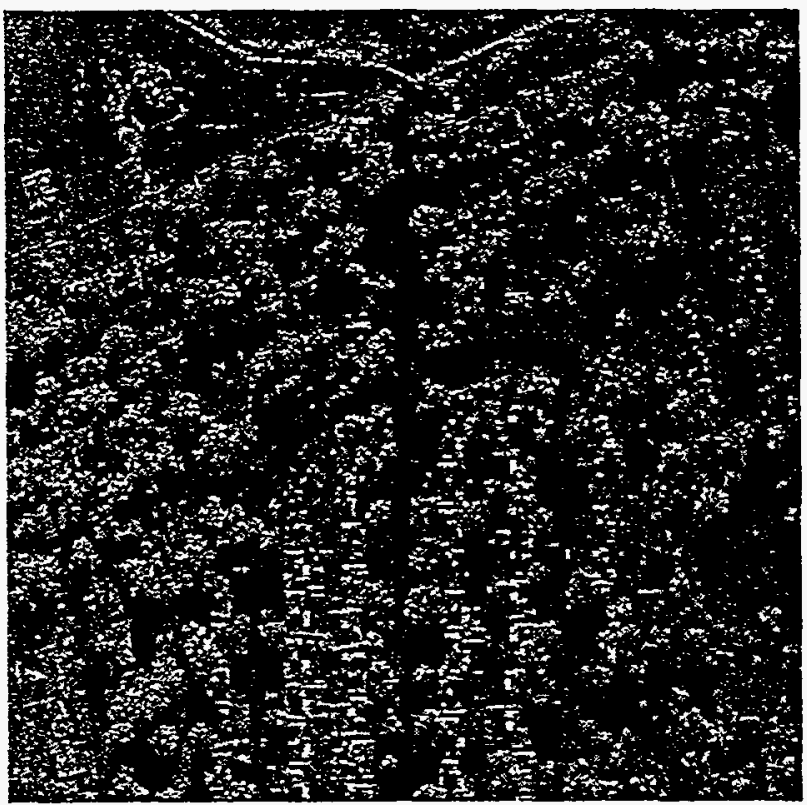

Figure 8: Houses.cmpx (1600x1600, $1 \mathrm{ft}$ res) 